\title{
Studying the Model of Free Harmonic Oscillations of Overhead Power Lines
}

\author{
Danil Aleksandrovich Yaroslavsky ${ }^{1}$, Van Vu Nguyen ${ }^{1}$, Marat Ferdinantovich Sadykov ${ }^{1}$, \\ Mikhail Petrovich Goryachev ${ }^{1}$, Dmitry Alekseevich Ivanov ${ }^{1}$, Tatyana Gennadievna Galieva ${ }^{1}$, \\ Yuliya Yurievna Vassunova ${ }^{1}$, Aleksandr Nikolaevich Tyurin ${ }^{2}$ \\ ${ }^{1}$ Kazan State Power Engineering University, Kazan, Russia \\ ${ }^{2}$ Kazan commissioning department of JSC "Tatelectromontazh", Russia
}

\begin{abstract}
This article is devoted to the importance of inspection of overhead power lines by detection of mechanical loads on conductors. A conductor under the action of forces caused by wind pressure swings like a pendulum. It is possible to determine conductor oscillations in space by inclinometer with high precision. While reconstructing conductor geometry in overhead power line span, it is possible to determine mechanical loads. Mechanical loads of overhead power line conductor are estimated using the developed model of free harmonic oscillations in span. The mathematical model of conductor is based on mathematical models of ideal cable and physical pendulum. Conductor is a physical pendulum, where a conductor acts as body, and fixed axle is presented by direct line passing through the conductor suspension points. The developed model determines conductor sag by oscillation period of conductor in span. The article discusses calculation algorithm of conductor sag for two cases: the conductor suspension points are at one height; the conductor suspension points are at different heights. Theoretical calculation is performed for the model of overhead power line span accompanied by estimation of sensitivity of the developed model and its error upon determination of conductor sag. Using the conductor sag, it is possible to reconstruct its geometry, thus, the mechanical loads of conductor. On the basis of initial geometrical parameters of overhead line span, it is possible to inspect its current state.
\end{abstract}

Key words: overhead power line, monitoring of overhead lines, sag, conductor oscillations, mechanical loads, conductor.

\section{INTRODUCTION}

Global energy market will grow up to 2035 due to increase in demand for energy in developing countries (being determined by growing industrialization and urbanization of
Asian and African regions as well as population increase) [1].

Energy is transferred from power stations or substations to consumer by overhead power lines (OPL). Total distance of OPL in Russia exceeds 2.8 million kilometers. Electrical grid facilities are getting outdated. The equipment wear reaches $70 \%$ [2]. However, due to increased demand for energy and implementation of new OPL segments, it becomes necessary to perform their complete inspection aiming at improvement of their operation.

OPL structural elements experience both horizontal (for instance, mechanical stresses in stringed wire/cable), and vertical loads (for instance, under own weight or under weight of wire/cable). Wires and cables are the elements of OPL, which most obviously exhibit variations of mechanical loads due to variation of wire/cable tension as a function of temperature and formation of ice deposits [3], as well as oscillations under wind blasts.

When mechanical load exceeds ultimate strength of OPL element, it can be damaged: breakage of conductor/cable, destruction or breakdown of isolator, phase fault due to swinging of conductors, etc.

Mechanical loads of OPL conductors are mainly estimated by two methods: direct measurements (by strain gauges) $[4,5$, 6] or indirect ones (inclinometry based on determination of conductor inclination angle using accelerometers) [7, 8, 9, 10]. In addition to numerous advantages, the weighing method is characterized by certain disadvantages, namely: necessity to modify line accessories and high costs [11]; unavailability of detection systems for OPL with the voltage of $35 \mathrm{kV}$ and below. In its turn, inclinometry is a reliable and straight method, it does not require to modify OPL design, though, it has measurement errors of inclination angle of sensor (accelerometer) and errors related with conversion of the inclination angle into conductor stringing force [7, 12].

Therefore, sufficiently precise localization of problematic segment of OPL or fault is required, since quite often travelling along OPL is difficult and the rate of fault elimination is directly related with incurred losses. Herewith, the quality improvement of OPL inspection should be accompanied by minimum expenses, that is, it is desirable to 
apply the hardware of existing OPL monitoring systems. The optimum variant is to develop monitoring system based on inclinometry, which combines reliability, high measurement accuracy of sensor deflection angle, as well as simplicity of installation of monitoring units with a set of sensors on OPL conductor.

A conductor swings under the action of forces caused by wind pressure. Using inclinometry, it is possible to detect conductor deflections from its equilibrium position in swinging plane with high accuracy. Therefore, a conductor in OPL span can be presented as a physical pendulum and can be included into the developed model of free harmonic oscillations of a conductor.

\section{METHODS}

A) Let us consider the model of free harmonic oscillations of conductor in span (without consideration for different heights of conductor suspension points).

Physical pendulum is a body, which under the action of force of gravity oscillates around fixed horizontal axis not passing through the center of gravity of the body. An OPL conductor can be considered as a physical pendulum with a conductor as the body and a line passing through the conductor suspension points as the fixed axle.

Let us present a conductor in a span as absolutely rigid isotropic structure with only one rotational degree of freedom with regard to the axis passing through the suspension points (Figure 1).

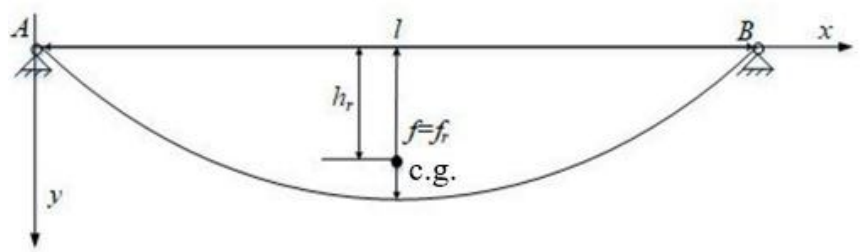

Figure 1: Model of OPL as a physical pendulum with designation of main geometrical parameters with suspension points at the same height: 1 is the horizontal distance between two adjacent points (length of span), $\mathrm{m}$; $\mathrm{f}$ is the difference in heights between the highest suspension point in the span and

the lowest point of conductor in the span (in this case, it equals to the conductor sag fr), $\mathrm{m}$; $\mathrm{hr}$ is the distance from the center of segment with tops in the suspension points A and B to the center of gravity of OPL, m.

Four variables are included into the equation of oscillation period of physical pendulum: $g$ is the acceleration of gravity, $h_{r}$ is the distance between the center of gravity (c. g.) and the axis of rotation, $m$ is the weight of body, $I$ is the moment of inertia with regard to the axis, around which oscillations take place:

$T-2 \pi \sqrt{\frac{I}{m g h_{r}}}$,
The inertia moment of conductor can be determined as follows:

$$
l-\int_{0}^{l} y^{2} d m_{w}
$$

where $y$ is the distance from the axis of rotation to infinitely small segment of conductor, $\mathrm{m}$.

The distance from the center of segment with tops in the conductor suspension points A and B to the center of gravity of OPL conductor along the $y$ axis is:

$$
h_{\mathrm{r}}-\frac{1}{m} \int_{0}^{1} y d m
$$

where the weight of infinitely small element of conductor is $d m=q d s ; q$ is the bulk weight of conductor $(\mathrm{kg}) ; d s$ is the length of infinitely small element of conductor, $\mathrm{m}$.

The length of infinitely small element of conductor upon displacement along the horizontal axis by the distance $x$ [13] is:

$$
d \varepsilon=\sqrt{1+y^{2 / 2}} d x
$$

where $\left|y^{\prime}\right|$ is the derivative with respect to $x$ axis.

At small sag angles, $\left|y^{\prime}\right|$ will be low as compared with one. Thus, Eq. (1) can be expanded into series and only two first terms can be retained:

$$
d s=\left(1+\frac{1}{2} y^{2}\right) d x .
$$

Without the difference in the height of OPL supports, the equation of conductor is as follows:

$$
y=\frac{1}{a}\left(\frac{l}{2} x-\frac{x^{2}}{2}\right), \text { where } a=\frac{i^{2}}{8 y^{\prime}}
$$

where $l$ is the span length, $\mathrm{m} ; f$ is the conductor sag, $\mathrm{m}$. Let us determine the distance from the center of swing axis to the center of swings.

$$
\begin{aligned}
& \boldsymbol{L}_{k}=\frac{I}{\mathrm{wh}_{\mathrm{r}}}=\frac{q \int_{0}^{l} y^{2}\left(1+\frac{y^{2}}{2}\right) d x}{q \int_{0}^{l} y\left(1+\frac{y^{2}}{2}\right) d x} \text { and after substitution of } \\
& \text { Then, }
\end{aligned}
$$
Eq. (3), integration and simplification, provided that $l \gg f$, the equation will be rewritten as follows:

$$
I_{k}-\frac{1}{m h_{r}}-\frac{4 f\left(7 l^{2}+0 f^{2}\right)}{7\left(5 l^{2}+0 f^{2}\right)} \approx \frac{4 f}{5} .
$$

Then, $T=2 \pi \sqrt{\frac{4 f}{5 g}}{ }^{s}$. Determining sag by the period, we obtain:

$$
f=\frac{b g]^{2}}{16 \pi^{2}} \approx 0,31 T^{2}
$$

where $T$ is the oscillation period, $\mathrm{s}$.

At oscillation angles higher than $15^{\circ}$, an adjustment is required so that the $f$ detection error is not higher than $0.5 \%$ [14]:

$$
f=\underset{16 M^{2}\left(1+\frac{1}{2^{2}} \sin ^{2} \frac{\theta_{M}}{2}+\frac{1 a^{2}}{2^{2} d^{2}} \sin \frac{d \theta_{M}}{2}+m\right)}{ }
$$

where $\theta_{M}$ is the maximum angular displacement of conductor (physical pendulum).

Therefore, the developed model of free harmonic oscillations of conductor in span with suspension points at 
one height makes it possible to determine conductor sag on the basis of the conductor oscillation period. More accurate determination of sag requires for maximum angular displacement of conductor in span.

B) Let us consider the model of free harmonic oscillations of conductor in span with consideration for different heights.

Let us present a conductor in span as absolutely rigid monolithic isotropic structure with only one rotational degree of freedom with regard to the axis passing through the suspension points located at different heights (Figure 2).

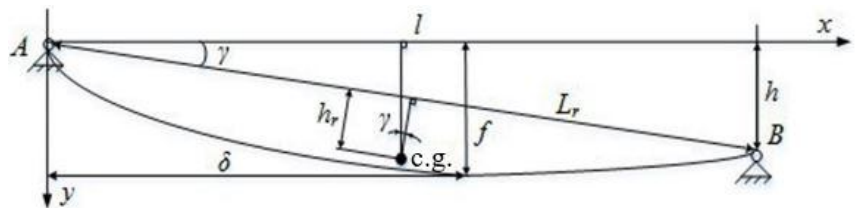

Figure 2: Model of OPL as a physical pendulum with designation of main geometrical parameters with suspension points at different height: $\mathrm{Lr}$ is the distance between suspension points, $\mathrm{m} ; \mathrm{l}$ is the length of span, $\mathrm{m}$; $\mathrm{f}$ is the difference in heights between the highest suspension point and the lowest point of conductor in the span, $\mathrm{m}$; $\mathrm{h}$ is the

difference in heights of suspension points, $\mathrm{m}$; $\mathrm{hr}$ is the

distance from the center of segment with tops in the suspension points A and B to the center of gravity of OPL, m; $\delta$ is the distance from the suspension point to the lowest point of conductor, $\mathrm{m}$; $\gamma$ is the acute angle between the horizontal line and the line passing through the conductor suspension

$$
\text { points, deg. }
$$

The moment of force acting on the conductor with regard to this axis of rotation passing through the conductor suspension points $\mathrm{A}$ and $\mathrm{B}$ is:

$\tau=-m g h_{r} \cos \gamma \sin \theta$.

where $\theta$ is the deflection angle of conductor from steady equilibrium in the swing plane. According to the Newton second law:

$$
\tau=I \frac{d^{2} \theta}{d t^{2}}
$$

At low oscillation angles, the equation of system balance is a harmonic equation:

The solution to this equation is:

$$
\frac{d^{2} \theta}{d t^{2}}+\frac{m g h_{r} \cos \gamma}{I}=0
$$

$$
\begin{gathered}
\theta=\theta_{M} \cos \left(\frac{2 \pi t}{T}+\varphi\right) \\
T=2 \pi \sqrt{\frac{1}{m g h_{r} m s \gamma}} .
\end{gathered}
$$

It can be seen in Fig. 2 that the height to the center of gravity is:

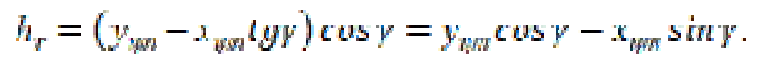

In integral form:

$$
\begin{gathered}
h_{r}=\frac{\cos \gamma}{m} \int_{n}^{i} y d n-\frac{\sin \gamma}{m} \int_{n}^{i} x d m, \\
d m-q d s,
\end{gathered}
$$

where $d s=\sqrt{1-y^{2}} d x$

$d s$ can be expressed in accordance with Eq. (2).

The inertia moment is as follows:

$$
I=\int_{0}^{l}(y \cos \gamma-x \sin \gamma)^{2} d m
$$

The distance from the axis of rotation to the swing center reduced to mathematical pendulum (suspension length) is as follows:

$$
L_{k}=\frac{I}{m h \cos \gamma}=\frac{\int_{0}^{l}(y \cos \gamma-x \sin \gamma)^{2}\left(1+\frac{1}{2} y^{\frac{12}{2}}\right) d x}{\cos \gamma \int_{0}^{l}(y \cos \gamma-x \sin \gamma)\left(1+\frac{1}{2} y^{2}\right) d x} .
$$

This equation considers for Eqs. 2, 6, 7 and the fact that $m$ and $q$ are contracted.

The equations of parabolic sag [13] are:

$$
y=\frac{1}{a}\left(\delta x-\frac{x^{2}}{2}\right), y^{\prime}=\frac{\delta-x}{n}, \delta=\frac{l}{2}+\frac{h}{l} a .
$$

While calculating the coefficient $a$, it is required to consider for adjustment according to Eq. (5) at oscillation angles $\theta_{M}$ higher than $15^{\circ}$.

Let us substitute Eq. (9) into Eq. (8) and take into account that $\cos \gamma={ }_{L_{i}} \cdot \sin \gamma={ }_{{ }_{i}}$, then after integration and a set of simplifications we obtain:

$$
L_{k}=\frac{l^{2}\left(l^{4}+56 u^{2} l^{2}\right)+28 u^{2} h^{n}}{14 a\left(l^{4}+40 a^{2} l^{2}+20 a^{2} h^{2}\right)}
$$

The distance from the axis of rotation to the swing center can be obtained on the basis of oscillation period:

$$
L_{k}=\frac{g T^{2}}{4 \pi^{2}}
$$

While solving Eq. (10) with regard to $a$, we conclude that it is a cubic equation with the only real root.

When the parabola coefficient $a$ is determined using Eq. (9), it is possible to calculate the parameter $f$, substituting $\delta$ instead of $x$ :

$$
f=\frac{\delta^{2}}{2 a}
$$

Equation (10) can be simplified by neglecting the terms $28 a^{2} h^{2}$ and $20 a^{2} h^{2}$ :

$$
L_{k}=\frac{l^{2}\left(i^{2}+56 a^{2}\right)}{\left.14 a a^{2}+40 a^{2}\right)^{n}}
$$

which would give the error less than $0.1 \%$, provided that $h$ $<l / 10$.

According to Eq. (11), it is possible to determine the distance from the axis of rotation to the swing center. Parameter $a$ is determined according to Eq. (12). Using the parameter $a$, it is possible to determine the conductor sag. 
Thus, it can be concluded that at $h<<L$, the swing center, hence, the period of free oscillations, does not depend on the difference in heights $h$, other span variables being the same. However, it should be mentioned that the parameter $a$ depends on the parameter $h$.

The vertical distance from the lowest point of curve to the axis of rotation (the conductor sag for the case with suspension points at different heights according to Electrical installation code [17]) is as follows:

$$
f_{p}=f-\delta t g y=f-\delta \frac{h}{l} .
$$

After determination of the center of gravity and drawing perpendicular to the axis of rotation through it, we determine the distance passing through the center of gravity from the curve (conductor) to the axis of rotation. It will be the maximum distance between the conductor and the axis of its rotation. After conversions, neglecting minor variables, we obtain a sag equivalent to the sag without difference in heights:

$$
f_{\varepsilon}=\frac{l^{2}}{3 a \sqrt{l^{2}+h^{2}}} \approx \frac{l^{2}}{8 a} .
$$

Therefore, the developed model of free harmonic oscillations of conductor in span considers for the difference in heights between the conductor suspension points. Herewith, it is possible to determine both the conductor sag and the maximum distance between the conductor and the axis of its rotation. The maximum distance between the conductor and the axis of its rotation allows to estimate the chance of crossing of the considered OPL conductors.

\section{RESULTS}

This section exemplifies numerical calculations of OPL span according to the model of free harmonic oscillations of conductor.

Let us execute theoretical calculations for OPL span model with the conductor, grade A-70, in the $50 \mathrm{~m}$ span upon variation of conductor oscillation period from $1.4 \mathrm{~s}$ to $2 \mathrm{~s}$.

Conductor sag is determined by Eq. (4) using the oscillation period. The calculated results are illustrated in Fig. 3.

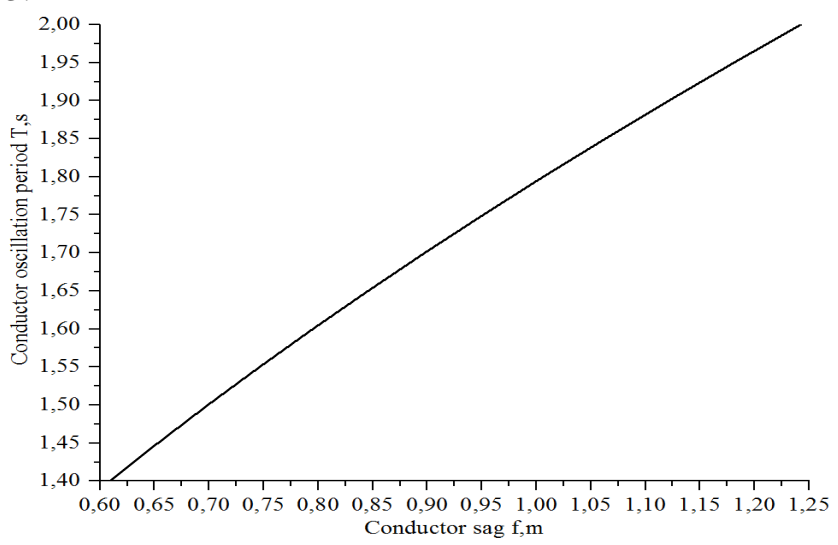

Figure 3: Oscillation period as a function of conductor sag.
It can be seen in Figure 3 that the sag increases with the conductor oscillation period.

Sensitivity of the model of free harmonic oscillations of conductor in span using the parameter $\mathrm{T}$ can be calculated as follows [15]:

$$
S_{T}=\frac{d f}{d T} .
$$

Then, the sensitivity of the developed model can be determined as follows:

$$
S_{T}=\frac{5 g T}{8 \pi^{2}} \approx 0_{s} 62 T .
$$

The sensitivity of the developed procedure upon variation of the conductor oscillation period from $1.4 \mathrm{~s}$ to $2 \mathrm{~s}$ varies from $0.870 \mathrm{~m} / \mathrm{s}$ to $1.243 \mathrm{~m} / \mathrm{s}$.

The error of determination of conductor sag is determined as follows:

$$
\Delta f=\frac{d f}{d T} \Delta T .
$$

While measuring the conductor oscillation period with discretization frequency of $1,000 \mathrm{~Hz}$, the conductor swing angle will be measured every millisecond. Therefore, the error of determination of conductor sag added by measurements for the considered case does not exceed 0.0012 $\mathrm{m}$, which is $0.097 \%$ of the maximum sag in this case.

\section{DISCUSSION}

The developed model of free harmonic oscillations of conductor allows to determine conductor sag (the most important variable for determination of current mechanical loads on OPL conductor [16]). Herewith, due to measurement of oscillation period of conductor, the sag is determined with high accuracy (the error of about $0.1 \%$ at equal height of suspension points). In addition, it is possible to determine the maximum distance between conductor and its pivot point, thus estimating the chance of crossing of OPL conductors.

Using the sag, it is possible to reconstruct the conductor geometry in span, that is, its length. On the basis of conductor initial tension and its current elongation, it is possible to estimate the tension [8] of conductor under current conditions. On the basis of tension, it is possible to estimate the danger of current mechanical loads on inspected OPL. Therefore, on the basis of initial geometrical parameters of OPL span and current conductor oscillation period, it is possible to inspect [16-19] its current state.

\section{CONCLUSION}

The model of free harmonic oscillations of conductor based on mathematical model of ideal cable and model of physical pendulum has been developed. This procedure allows to determine conductor sag by its oscillations with regard to its equilibrium position. Herewith, the accuracy of sag determination depends on frequency of measurements of conductor position (increase in the frequency of measurement discretization increases the determination accuracy of 
conductor sag). After determination of conductor sag, it is possible to transfer to determination of mechanical loads of OPL conductors, thus estimating their current state.

However, the developed model should be experimentally verified, at present only theoretical possibility to determine conductor sag by its oscillation period has been proved.

In addition, it will be required to estimate the influence of wind blast on oscillations of OPL conductor.

The model of free harmonic oscillations of conductor is the result of studies published by the authors. In order to develop and implement the measurement procedure of mechanical loads of OPL conductors using the model of free harmonic oscillations of conductor, it is proposed to use previously developed solution in the form of monitoring instrument of OPL mechanical variables.

\section{ACKNOWLEDGEMENT}

This work was supported by the Ministry of Science and Higher Education of the Russian Federation, project No. 075-15-2020-172.

\section{REFERENCES}

1. Action PLAN ("road map") "Energinet" of the National technological initiative. INNOVATIONS in the fuel and energy sector project of the Ministry of energy of Russia. Retrieved from: https://in.minenergo.gov.ru/energynet/docs/DK_energy net.pdf

2. G. Bokov. Technical re-equipment of Russian electric networks. How much can it cost? Electrical Engineering News no. 2(14), 2002.

3. E.I. Satsuk. Software and technical means of monitoring overhead power transmission lines and power system management in extreme weather conditions: dis. ... doctor of technical Sciences. Novocherkassk, 2011.

4. S.V. Mortician, D.S. Kim. Information-measuring system of control of ice deposits on the wires of power lines. Energy and resource saving: industry and transport no. 2, pp. 7-11, 2017.

5. R.G. Minullin. Modern methods of detecting ice on overhead power lines part 1 . Methods of forecasting and weighing wires. News of higher educational institutions. Energy problems no. 7-8, pp. 68-78, 2013.

6. D.A. Ivanov, A.V. Golenishchev-Kutuzov, D.A. Yaroslavsky, M.F. Sadykov. Portable complex for remote control of high-voltage insulators using wireless data collection and transmission module. ARPN Journal of Engineering and Applied Sciences vol. 13 no. 6, pp. 2358-2362, 2018.

7. V.G. Makarov, A.I. Fedotov, R.Sh. Basyrov, G.V. Vagapov. Modeling of an overhead power transmission line in the Matlab/Simulink package. Bulletin of the Kazan technological University vol. 20 no. 12, pp. 93-96, 2017.
8. D.A. Yaroslavsky, M.F. Sadykov, D.A. Ivanov, M.P. Goryachev, A.B. Konov. Methodology of ice coating monitoring on overhead transmission lines considering misalignment using wireless communication channel sensors. ARPN Journal of Engineering and Applied Sciences vol. 12 no. 22, pp. 6479-6482, 2017.

9. A. Fedotov, S. Kurth, S. Voigt, G. Vagapov. A concept for an ice detection system on overhead power lines, theory and practical results. Proceedings of the 9th International Scientific Symposium on Electrical Power Engineering. Elektroenergetika, pp. 297-300, 2017.

10. T. Otto. Integrated Microsystems for Smart Applications. Sensors and Materials vol. 30 no. 4, pp. 767-778, 2018. https://doi.org/10.18494/SAM.2018.1797

11. A.S. Zasypkin, A.N. Shchurov, A.D. Teterin. Application of icy load sensors and sensors of longitudinal tension of Overhead line wires for assessing the danger of icy conditions. University news. North Caucasus region. Series: Technical Sciences no. 2(198), 2018.

12. V.S. Petrov, T.I. Dubrovskaya. Mechanical calculation of wires and cables of overhead lines as a basis for calculating the reliability of structures. Online journal Naukovedenie vol. 7 no. 6(31), pp. 11-13, 2015.

13. D.R. Merkin. Introduction to the mechanics of flexible thread. Moscow: Nauka, 1980.

14. D. Giancoli. Physics: In 2 vols. Moscow: Mir, 1989.

15. V.Ya. Andreev, E.M. Antonyuk, E.M. Dushin. Fundamentals of Metrology and electrical measurements. Leningrad: Energimidt. Leningr. otd-nie, 1987.

16. Overhead power transmission lines with a voltage higher than $1 \mathrm{kV}$. M, JSC "VNIIE". PUE. ed. 7-e. Chapter 2.5, 2003.

17. M.P. Goryachev, M.F. Sadykov, D.A. Yaroslavskiy. Method for control the mechanical parameters of overhead power lines based on improved inclinometry. Power engineering: research, equipment, technology vol. 21 no. 3, pp. 160-171, 2019.

18. M.F. Sadykov, M.P. Goryachev, D.A. Yaroslavsky, D.A. Ivanov, I.M. Koryshkin. RF patent №185311. A device for operational monitoring of the technical condition of high-voltage power lines. Russian Patent No. 2018120028, 2018. Bull. Number 34.

19. A.V.Golenishchev-Kutuzov,V.A.Golenishchev-Kutuzov , D.A. Ivanov, G.D. Mardanov, A.V. Semennikov. Integrated noncontact diagnostics of the operable condition of high-voltage insulators. Russian Journal of Nondestructive Testing vol. 55 no. 8, pp. 596-602, 2019. https://doi.org/10.1134/S1061830919080060 